\title{
Application of the Artificial Bee Colony Based on Boltzmann in Geometric Constraint Problem
}

\author{
Wenhui Li ${ }^{12}$, Mingyu Sun ${ }^{3}$ \\ Department of Computer Science and Technology, Jilin University, Changchun, 130012, China \\ E-mail: Iiwhejlu.edu.cn; sunmingyu370@sohu.com
}

\section{Chunhong Cao}

College of Information Science and Engineering ,Northeastern University,Shenyang, 110819, China

E-mail: caochunhong@mail.neu.edu.cn

\section{Mingyan Sun, Honghao Shen}

West New Economic-Technological Development, Zone Construction Industries Co., Ltd, Chang Chun, 130012, China

E-mail: smy_12130foxmail.com; shenhh1260163.com

\section{Baoliang Mu}

College of Software Normal University, Shenyang, 110034, China

E-mail: mublesynu. edu. cn

Solving the geometric constraint problem is substantially equivalent to solving a system of nonlinear equations; furthermore, we can transform a geometric constraint problem to an optimization problem. By using Artificial Bee Colony, the optimization problem can be solved. The Artificial Bee Colony (ABC) algorithm is an optimization algorithm and mainly on the basis of the intelligent act of honeybees. In this paper, an improved $\mathrm{ABC}$ algorithm is proposed (also called BABC) based on the Boltzmann selection mechanism and used to solve the geometric constraint. BABC algorithm makes the initial group symmetrically. Instead of roulette, this method uses Boltzmann selection mechanism to avoid premature. Experiment results indicate that the $\mathrm{BABC}$ has effectively improved the solution efficiency of the geometric constraint problems and better converge to the optimal solution than the traditional $\mathrm{ABC}$ algorithms.

CENet2015

12-13 September 2015

Shanghai, China

\section{${ }^{1}$ Speaker}

${ }^{2}$ This work is supported by "Grant from Jilin Planned Projects for Science Technology Development" (Grant No.: 20140101181JC and 20120305), the "National Natural Science Fund" (Project No.: 61300096) and the "Fundamental Research Funds for the Central Universities" (Project No.: N130404013).

${ }^{3}$ Correspongding Author 


\section{Introduction}

The methods of solving the geometric constraint problems mainly include algebraic-based method, rule-based method and graph-based method [1]. One geometric constraint expresses a kind of relation that can be satisfied between geometric primitives. When the users define a set of geometric constraints, the solver will automatically satisfy them by selecting proper states upon modifying some parameters.

The ultimate goal of solving a geometric constraint problem is to obtain the specific coordinates of each location in the geometry. Thus we may transform a geometric constraint problem to an optimization problem, but some classical algorithms such as the Ant colony algorithm and the partical swarm optimization (PSO) algorithm of objective functions and constraints have certain requirements. Usually, the convergence speed of the ant colony algorithm is slow and it is likely to be subject to stagnation and trap in local optimum, etc.; besides, the PSO algorithm is also prone to be subject to trap in local optimum and even shock. In this work, we use an improved $\mathrm{ABC}$ algorithm. In the course of searching the basic $\mathrm{ABC}$ algorithm, we does not use external information, only to use fitness function as the basis of evolution and form the "generate + test" in respect of characteristics of the artificial intelligence technology. The $\mathrm{ABC}$ algorithm has simple operation, less control parameters, higher precision search of the characteristics and strong robustness [2-3]. In recent years, a new bee colony algorithm which uses random search method was put forward in the field of optimization [4-10]. The main feature of $\mathrm{ABC}$ is characterized by the local individual search behavior of various work bees, ultimately, the global optimum manipulation in groups comes to the fore; besides, it has a faster convergence rate; nevertheless, when the Pre-optimization problem has many local extreme points, it only has one global minimum point and there is a near global optimal cirque. The unimproved $\mathrm{ABC}$ algorithm is not satisfactory. As to the problem of the $\mathrm{ABC}$ algorithm, we introduce a novel $\mathrm{ABC}$ algorithm on the basis of the selection mechanism of Boltzmann [11] (BABC algorithm). On the basis of a series of experiments and results, it shows that the improved algorithm $\mathrm{BABC}$ highlights sound ability of finding the optimal solution.

\section{Geometric Constraint Solving}

From the point of view of artificial intelligence [12-13], the nature of the design problem is a constraint satisfaction problem. Among many design constraints, the geometric constraint is the most basic and it is basic for the expression of other design constraints; moreover, the priority solves issues among the constraint management and solution techniques. The ultimate goal of solving a geometric constraint problem is to obtain the actual coordinates of each location in the geometry.

The geometric constraint problem may be represented as $G C=(E, C)$ [14], where, $E=\left(e_{1}, e_{2}\right.$, $\left.\ldots, e_{n}\right)$, represents the geometric primitives, such as point, line and circle, etc; $C=\left(c_{1}, c_{2}, \ldots, c_{m}\right)$, where $c_{i}$ is the constraint between these geometric primitives. Generally, one geometric constraint is represented by a nonlinear algebraic equation, so a geometric constraint problem can be expressed as follows:

$$
\begin{gathered}
f_{1}\left(x_{0}, x_{1}, x_{2}, \ldots, x_{n}\right)=0 \\
\ldots \\
f_{m}\left(x_{0}, x_{1}, x_{2}, \ldots, x_{n}\right)=0
\end{gathered}
$$

$X=\left(x_{0}, x_{1}, \ldots, x_{n}\right)$, where, $x_{i}$ is the value of some parameters of the geometric elements $e_{i}$, for example, the two-dimensional point may be represented as $\left(x_{1}, x_{2}\right)$. The constraint solution is exactly to find the $X$ that satisfies (2.1). 


$$
F\left(X_{i}\right)=\sum_{i=1}^{m}\left|f_{i}\right|
$$

Obviously, if $F\left(X_{j}\right)=0$ can be satisfied by $X_{j}$, the above Formula (2.1) can be satisfied by $X_{j}$. Thus an optimization problem can be obtained by transforming the geometric constraint problem and we only need to calculate $\min \left(F\left(X_{j}\right)\right)<$, where, is a specific threshold. In order to improve the BABC speed, the absolute value of $f_{i}$ is adopted instead of the square sum to represent the constraint equations. We can realize from Formula (2.2) and by calculating $\min \left(F\left(X_{j}\right)\right)<$ by $\mathrm{BABC}$ that it is unnecessary $m=n$, thus the BABC algorithm may also handle the underconstrained and over-constrained problem.

\section{Basic Principles of ABC Algorithm}

In the $\mathrm{ABC}$ algorithm, a potential solution of optimization problem is represented by the location of a food source, therein the amount of the nectar represents the quality of the obtained solution (fitness). The number of the leading bees equals to the number of the following bees and it also equals to the number of solution. Firstly, the algorithm creates an initial population which includes $S N$ solutions (food sources). Every solution $x_{i}(i=1,2 \ldots, S N)$ represents a $D$ dimensional vector; secondly, the bees find the food sources by making a circularly search. The number of the cycle is MCN. At the beginning, the leading bee finds the corresponding food source by making a neighborhood search and chooses the food sources, in which the nectar amount is high. The entire leading bees transmit the information to the following bees in the dance area after their finding of the food source. The following bees will choose the food source in accordance with the probability and based on the information as gained. It will be of higher probability that the food source with more nectar will be chosen; thirdly, the following bees will also make a neighborhood search and choose a better solution. The food source is choosen by the following bees in accordance with the probability $P_{i}$, the expression of which is

$$
p_{i}=\frac{f_{i}}{\sum_{n=1}^{S N} f_{n}}
$$

In Equation (3.1), $f_{i}$ represents the fitness of the $i^{\text {th }}$ solution.

The leading bee and the following bees make the neighborhood search according to the following expression

$$
v_{i j}=x_{i j}+R_{i j}\left(x_{i j}-x_{k j}\right)
$$

where, $k\{1,2, \ldots, S N\}, j\{1,2, \ldots, D\}$, and they can be chosen randomly; but $k$ can't equal to $i$. $R_{i j}$ is a random number between -1 and 1 . This step can be appropriately reduced.

If the $\mathrm{ABC}$ algorithm can not improve a solution after limit cycles, this solution will be discarded; at meanwhile, "limit" is regarded as an important control parameter. Suppose the solution be discarded as $x_{i}$; then the detecting bees will generate a new solution to replace $x_{i}$ by means of the follow expression.

$$
x_{i}^{j}=x_{\min }^{j}+\operatorname{rand}(0,1)\left(x_{\max }^{j}-x_{\min }^{j}\right)
$$




\section{Bee Colony Algorithm Based on Boltzmann}

The $\mathrm{ABC}$ algorithm is primarily characterized by the individual local search behavior of various work bees, ultimately, the global optimum manipulation in groups comes to the fore and has a faster convergence rate; but when the pre-optimization problem has many local extreme points, there will be only one global minimum point and a near global optimal valley; and the unimproved $\mathrm{ABC}$ algorithm is not satisfactory. As to such problem of the $\mathrm{ABC}$ algorithm, this paper introduces a novel $\mathrm{ABC}$ algorithm which is mainly based on the selection mechanism of Boltzmann, that is, BABC algorithm.

\subsection{Improve the Selection Mechanism}

In the above algorithm, the following bees choose the food source in accordance with the probability based on the method of roulette, but the selection method of roulette is likely to result in the decrease of population diversity and the algorithm will converge prematurely. As a result, in the course of searching the optimal solution, different selection pressures are required on different stages with less pressure on the early stage of selection. We hope that the poor individuals can also have a chance of survival, which will make the group to maintain high diversity and greater selection pressure on the late stages, and we hope the algorithm to narrow the search area and accelerate the change rate of the current optimal solution. In order to dynamically adjust the selection pressure in the course of searching optimal solution based on analysis of features of the search mechanism of the existing colony algorithm, we introduce the Boltzmann selection strategy into the search process.

In the course of machine learning, adaptive control and other fields, Boltzmann selection strategy has been widely used by the search algorithm. The selective probability that the following bees used to choose the food source is

$$
P_{i}=\frac{\exp \left(\frac{f_{i}}{T}\right)}{\sum_{i=1}^{S N} \exp \left(\frac{f_{i}}{T}\right)} T=T_{0}\left(0.99^{c-1}\right)
$$

Where, $f_{i}$ is the fitness of the $i$ th individual, $c$ is the cycle number, $t$ is the temperature and $T_{0}$ is the initial temperature.

\subsection{Improve the Selection Mechanism}

In the BABC algorithm, there are mainly four control parameters, including $S N$-the food source number, limit, $M C N$-the maximum cycle number and the initial temperature $T_{0}$, in which, $S N$ equals to the number of leading bees and the number of the following bees. The algorithm steps are shown as follows.

Step 01: generate the set of the initial solution $x_{i j}, i=1,2, \ldots, S N$ and $j=1,2, \ldots, D$;

Step 02: compute the fitness of every solution $x_{i j}$;

Step 03: set cycle $=1$; (outer loop)

Step 04: set s=1; (inner loop)

Step 05: the leading bee makes the neighborhood search according to Equation (3.2) and generates the new solution $v_{i j}$, and then computes its fitness;

Step 06: if the fitness of $v_{i j}$ is larger than $x_{i j}$, then $x_{i j}=v_{i j}$; else $x_{i j}$ will not change;

Step 07: compute the fitness of $x_{i j}$ while computing the probability of $p_{i j}$ based on Equation (4.1);

Step 08: the following bees choose the food source based on $P_{i j}$, and make a neighb- orhood search for the corresponding food source, then generate a new solu- tion $v_{i j}$ and compute its fitness;

Step 09: it is the same to Step 6; 
Step 10: record the best solution so far;

Step 11: $s=s^{+}$;

Step 12: cycle $=$ cycle+1;

Step 13: if $s<$ limit, then go to Step 5;

Step 14: determine whether there is a solution that should be discarded after cycles for limit times. If there is, detect the bees generating a new solution $x_{i j}$ based on Equation (3.3);

Step 15: if cycle $<M C N$, go to Step 4;

\section{Application Example and Result Analysis}

The development environment of experiment is MATLAB6.5. Run under Pentium4/2.8G hardware conditions. There are four parameters to be set in BABC algorithm: the population size $\mathrm{SN}$, the search algebra $\mathrm{MCN}$, the limit and the initial temperature $\mathrm{T}_{0}$.

(1) Sphere Function

$$
f_{1}(X)=\sum_{i=1}^{n} x_{i}^{2} \quad-5.12 \leq x_{i} \leq 5.12 \quad(i=1,2, \ldots, n)
$$

(2) Rosenbrock Function

$$
f_{2}(X)=\sum_{i=1}^{n}\left[100\left(x_{i+1}-x_{i}^{2}\right)+\left(x_{i}-1\right)^{2}\right]-2.048 \leq x_{i} \leq 2.048 \quad(i=1,2, \ldots, n)
$$

(3) Rastrigin Function

$$
f_{3}(X)=\sum_{i=1}^{n}\left(x_{i}^{2}-10 \cos (2 \pi x)+10\right) \quad-5.12 \leq x_{i} \leq 5.12 \quad(i=1,2, \ldots, n)
$$

where, $f_{1}(X)$ is a simple unimodal function and reaches the minimum at $(0,0,0)$; the dimensional rosenbrok function $f_{2}(X)$ is a non-convex function and reaches the minimum point at $(1,1) ; f_{3}(X)$ is called Rastrigin function and reaches a global minimum when $x_{i}=0, i=1,2, \ldots, n$. The function has about $10 \mathrm{n}$ local minimum points in $-5.12 \leq x_{i} \leq 5.12(i=1,2, \ldots, n)$.

Function $f_{1}(X), 30$ dimensions. The parameter set of $\mathrm{ABC}$ algorithm is: population size $S N=50$; search algebra $M C N=200$; limit $=50$. The results of continuous operations for 50 times are: the average optimal solution of $1.249 \mathrm{e}-13$ and the success rate of $100 \%$.

Function $f_{2}(X), 30$ dimensions. The parameter set of algorithm is: $S N=50 ; M C N=300$; limit $=50 ; \mathrm{T}=100$. The results of continuous operation for 50 times are: the average optimal solution of $1.009 \mathrm{e}-7$ and the success rate of $100 \%$.

Function $f_{3}(X), 30$ dimensions. parameter set of algorithm is: $S N=100 ; M C N=500$; limit= 50. The results of continuous operation for 50 times are: the average optimal solution of $5.329 \mathrm{e}-$ 13 and the success rate of $100 \%$.

Table 1 shows the comparison among the experimental results of the algorithm BABC with the standard ABC algorithm and the standard PSO algorithm. From Table 1, ABC algorithm has good ability of optimization when there are less local extreme points in the search space; as to the complex functions, the optimized results of ABC algorithm and PSO algorithm are not ideal. It's easy to fall into local optima. But the improved BABC algorithm for the test function, especially for the complex multimodal function, highlights better optimization results than those of previous two methods because the BABC algorithm contains both the deterministic and and stochastic search factors, which can exhibit strong and optimized performance in dealing with multi-dimensional complex functions. 


\begin{tabular}{|l|l|l|l|l|l|}
\hline \multirow{4}{*}{ Best value } & Target fuction & $\begin{array}{l}\text { Dimensi } \\
\text { on }\end{array}$ & BABC & ABC & PSO \\
\cline { 2 - 6 } & Sphere & 30 & $3.594 \mathrm{e}-14$ & $2.727 \mathrm{e}-13$ & $1.398 \mathrm{e}-2$ \\
\cline { 2 - 6 } & Rosenbrock & 30 & $8.258 \mathrm{e}-15$ & 0.099 & 50.352 \\
\cline { 2 - 6 } & Rastrigin & 30 & 0 & $2.576 \mathrm{e}-16$ & 20.267 \\
\hline \multirow{5}{*}{ Worst value } & & & BABC & ABC & PSO \\
\cline { 2 - 6 } & Sphere & 30 & $7.564 \mathrm{e}-13$ & $2.657 \mathrm{e}-12$ & $4.609 \mathrm{e}-2$ \\
\cline { 2 - 6 } & Rosenbrock & 30 & $1.333 \mathrm{e}-6$ & 10.275 & 146.725 \\
\cline { 2 - 6 } & Rastrigin & 30 & $1.154 \mathrm{e}-12$ & $2.952 \mathrm{e}-11$ & 106.037 \\
\hline \multirow{5}{*}{ Average value } & & & BABC & ABC & PSO \\
\cline { 2 - 6 } & Sphere & 30 & $1.249 \mathrm{e}-13$ & $7.663 \mathrm{e}-13$ & $3.986 \mathrm{e}-2$ \\
\cline { 2 - 6 } & Rosenbrock & 30 & $1.009 \mathrm{e}-7$ & 4.761 & 98.265 \\
\cline { 2 - 6 } & Rastrigin & 30 & $5.329 \mathrm{e}-13$ & $2.199 \mathrm{e}-12$ & 48.713 \\
\hline
\end{tabular}

Table 1: Comparison of Results of $\mathrm{BABC}$ with $\mathrm{ABC}$ and $\mathrm{PSO}$

(a) and (b) in Fig. 1 refer to drafts in engineering design. (b) is automatically generated upon modification of several specific sizes parameters by performing the BABC algorithm. It could be seen from the example of Fig. 1 that once a set of geometric constraints are designated by the $\mathrm{BABC}$ algorithm, the constraints can be satisfied by the solver by means of choosing proper state upon modification of some parameters.

Now we compare the searching capability of the traditional genetic algorithm (GA), the traditional PSO algorithm and the BABC algorithm in case of solving a geometric constraint problem. Table 2 as follows summarizes the searching capability of three different algorithms.

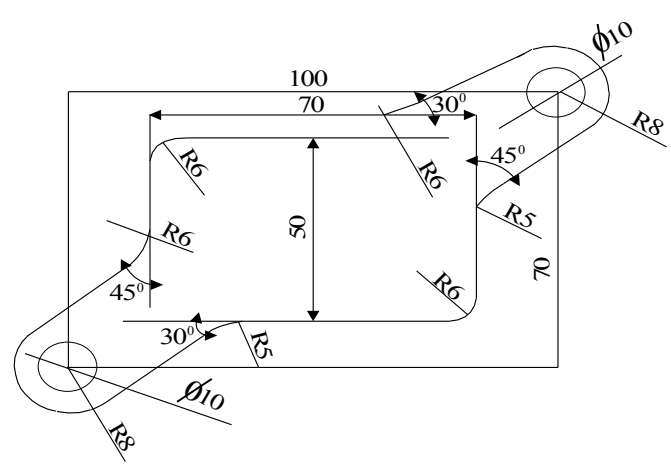

(a) A Design Instance

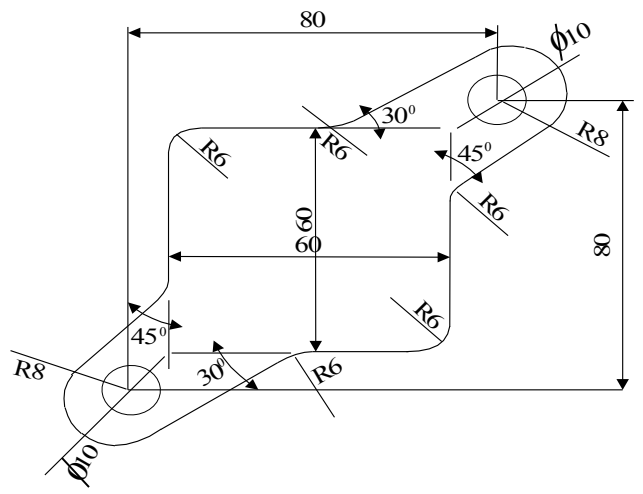

(b) Solving Result

Figure 1: A Design Instance of Geometric Constraint Problem 


\begin{tabular}{|l|l|l|l|}
\hline Algorithm & $\begin{array}{l}\text { Generation of } \\
\text { evolution }\end{array}$ & $\begin{array}{l}\text { Time of occupying } \\
\text { CPU(s) }\end{array}$ & $\begin{array}{l}\text { The generation of } \\
\text { appearing optimal solution }\end{array}$ \\
\hline GA & 500 & $48 \mathrm{~h}$ & 488 \\
\hline PSO & 20 & 20 & 15 \\
\hline BABC & 14 & 10 & 10 \\
\hline
\end{tabular}

Table 2: Comparison of GA, PSO and BABC

It could be seen from Table 2 that although the three algorithms can uniformly obtain the optimal solution in limited generations, the solving efficiency of the BABC algorithm is higher than that of the others.

\section{Conclusion}

Geometric constraint solving is one of key techniques of modern parametric design because the solving quality is directly related to the pros and cons of a parametric design system. In this paper, in accordance with the fact that we can transform a geometric constraint problem to an optimization problem corresponding to the constraint equations of the problem, we introduce a $\mathrm{BABC}$ algorithm based on the selection mechanism of Boltzmann so as to solve the constraint problem. Experiment results show that the improved algorithm BABC features good ability in terms of solving the constraint problem. As a new kind of swarm intelligence evolutionary algorithm, the $\mathrm{ABC}$ algorithm has global convergence and can meet the range for wide use; besides, it needs fewer parameters and highlights the parallelism in nature and other advantages.

\section{References}

[1] B. Yuan. The Research and Implement of Geometric Constraint Solving [D]. Tsinghua University, Beijing, 1999. (In Chinese)

[2] S. M. CHEN, A. Sarosh, Y. F. Dong. Simulated anealing based artificial bee colony algorithm forglobal numerical optimizaiton [J]. Applied Mathematics and Computations, 2012, 219(8) : 35753589.

[3] S. K. Mustafa, G. Mesut. A recombination-based hybridization of particle swarm optimizationand artificial bee colony algorithm for continuous optimization problems [J]. Applied Soft computing, 2013, 4(13): 2188- 2203.

[4] D. Teodorovic, M. Dell' Orco. Bee colony optimization-a cooperative learning approach to complex transportation problems [C]. Proceedings of the 10th EWGT Meeting, Advanced OR and AI Methods in Transportation, Poznan, 13-16 September 2005.

[5] H. Drias, S. Sadeg, S. Yahi. Cooperative bees swarm for solving the maximum weighted satisfiability problem, computational intelligence and bioinspired systems $[\mathrm{C}]$. Proceedings of the 8th International Workshop on Artificial Neural Networks, IWANN 2005, Vilanova ila Geltr, Barcelona, Spain, 8-10 June 2005.

[6] H. A. Abbass. Marriage in honey-bee optimization (MBO): a haplometrosis polyginous swarming approach $[\mathrm{C}]$. The Congress on Evolutionary Computation, IEEE, Seoul, Korea, May 2001: 207-214.

[7] H. A. Abbass. A monogenous $M B O$ approach to satisfiability [C]. Proceeding of the International Conference on Computational Intelligence for Modeling, Control and Automation, Australia and IEEE ACT Section, Las Vegas, NV, USA, 2001: 113-122.

[8] X. S. Yang. Engineering optimizations via nature-inspired virtual bee algorithms [C]. Lecture Notes in Computer Science. Springer, Berlin, Heidelberg, 2005: 317-323. 
[9] D. Karaboga.An idea based on honey bee swarm for numerical optimization, Technical ReportTR06 [R]. Erciyes University, Engineering Faculty, Computer Engineering Department, 2005.

[10] Y. J. Wang. Research on the Improvement and Application of Artificial Bee Colony Algorithm [D]. Harbin Engineering University, CNKI, 2013. (In Chinese)

[11] H. J. Ding, Q. X. Feng. Artificial bee colony algorithm based on Boltzmann selection policy [J]. Computer Engineering and Applications, 2009, 45(31): 53-55.

[12] J. S. Fu, Z. X. Cai, G. Y. Xu. Artificial Intelligence and its Applications [M]. Beijing: Higher Education Press, 1987: 15-28. (In Chinese)

[13] J. Zhou, J. Z. Cha, R. B. Xiao. Intelligent Design [M]. Beijing: Higher Education Press, 1998: 112. (In Chinese)

[14] S. L. Liu, M. Tang, J. X. Dong. Two spatial constraint solving algorithms [J]. Journal of Computer-Aided Design and Computer Graphic, 2003, 15(8): 1011-1029. (In Chinese) 